\title{
Predictors of Absent High-grade Cervical Intraepithelial Neoplasia (CIN) in Loop Electrosurgical Excision Procedure Specimens of Patients with Colposcopic Directed Biopsy-Confirmed High-Grade CIN
}

\author{
Yenrudee Poomtavorn ${ }^{1 *}$, Chamnan Tanprasertkul², Araya Sammor ${ }^{3}$, Komsun \\ Suwannarurk ${ }^{1}$, Yuthadej Thaweekul ${ }^{1}$
}

\begin{abstract}
Objective: To determine predictors of having cervical intraepithelial neoplasia (CIN) 1 or less in loop electrosurgical excision procedure (LEEP) specimens of patients with colposcopic directed biopsy-confirmed CIN 2-3. Methods: Two hundred and eighty patients with colposcopic directed biopsy-confirmed CIN 2-3 who subsequently underwent LEEP were enrolled in the retrospective study. Related clinical data were collected to determine the predictors of CIN 1 or less in LEEP specimens. Results: CIN 1 or less in LEEP specimens was found in 71 (25.4\%) of 280 patients. Multivariate logistic regression analyses demonstrated that nulliparity [OR $(95 \% \mathrm{CI})=3.375(1.245-9.150)$ ], low grade Papanicolaou (Pap) results [OR $(95 \%$ CI) $=6.410(2.877-14.280)]$ and low grade colposcopic impression [OR $(95 \% \mathrm{CI})=16.506(5.844-46.632)]$ were significant risk factors of having CIN 1 or less in LEEP specimens. Neither persistent nor recurrent CIN 2-3 was detected in 71 patients who had CIN 1 or less in LEEP specimens. However, persistent or recurrent CIN 2-3 developed in 3 out of 209 (1.4\%) patients with CIN 2-3 found in LEEP specimens. Conclusion: Approximately $25 \%$ of patients with CIN 2-3 in colposcopic directed biopsy specimens had CIN 1 or less found in LEEP specimens. Predicting factors of having CIN 1 or less in LEEP specimens were nulliparity, low grade Pap results and low grade colposcopic impression.
\end{abstract}

Keywords: Loop electrosurgical excision procedure- cervical intraepithelial neoplasia- colposcopy

Asian Pac J Cancer Prev, 20 (3), 849-854

\section{Introduction}

Early detection and treatment of high grade cervical intraepithelial neoplasia (CIN 2-3) is known to prevent the development of cervical cancer (Pinto and Crum, 2000; Massad et al., 2013). Management options for CIN 2-3 are ablation and excision in which both techniques have a comparable efficacy (Massad et al., 2013). However, the excisional method offers more advantages, including obtaining a specimen for pathological assessment and indicating a complete removal of lesion (Kyrgiou et al., 2006; Soutter et al., 2006; Martin-Hirsch et al., 2013). Many techniques of the excisional method are available, including loop electrosurgical excision procedure (LEEP), laser excision and cold-knife conization (Martin-Hirsch et al., 2013). LEEP is preferably used for treating CIN 2-3 lesions because it is safe, cost-effective and practical to perform under local anesthesia in an outpatient setting (Eduardo et al., 1996).
Pathological findings of LEEP specimens are often correlated with the results of colposcopic directed biopsy-confirmed CIN 2-3. However, some LEEP specimens of patients with colposcopic directed biopsy-confirmed CIN 2-3 reveal only CIN 1 or less. Previous studies have reported that there were $14-24 \%$ of patients with colposcopic directed biopsy-confirmed CIN 2-3 had CIN 1 or less in LEEP specimens (Ryu et al., 2010; Witt et al., 2012; Rodriguez-Manfredi et al., 2013; Giannella et al., 2015; Nam et al., 2015; Zhang et al., 2015). Therefore, routine treatment of all patients with biopsy-confirmed CIN 2-3 by using LEEP may result in overtreatment. Although LEEP is a safe excision procedure, it may carry complications, including bleeding, infection, incompetent cervix and cervical stenosis. These complications may result in an increased risk of future pregnancy problems.

The objectives of this study were to determine the frequency of CIN 1 or less in LEEP specimens of

${ }^{1}$ Gynecologic Oncology Unit, ${ }^{2}$ Reproductive Medicine Unit, Department of Obstetrics and Gynecology, ${ }^{3}$ Department of Pathology and Forensic Medicine, Faculty of Medicine, Thammasat University Hospital, Pathumthani, Thailand. *For Correspondence: pyenrudee@gmail.com 
women with colposcopic directed biopsy-confirmed CIN 2-3 and to evaluate the predicting factors of having CIN 1 or less in these LEEP specimens.

\section{Materials and Methods}

This retrospective study was undertaken at the Department of Obstetrics and Gynecology, Faculty of Medicine, Thammasat University Hospital (a tertiary hospital), Thailand. The study was approved by the Institutional Review Board of the Faculty of Medicine, Thammasat University Hospital and conformed the Enhancing the QUAlity and Transparency Of health Research (EQUATOR) network guidelines. Women with abnormal Papanicolaou (Pap) test who had been diagnosed with CIN 2-3 by colposcopic directed biopsy and subsequently underwent LEEP between January 2012 and December 2017 were included in the study. Pregnant women, patients who had a history of precancerous or cancerous lesion of the cervix, patients who had previous cervical surgery or hysterectomy and patients who had hysterectomy within 3 months after LEEP were excluded.

All related clinical data were collected. The collected data included demographic data, Pap test results, colposcopic findings, pathological diagnoses of colposcopic directed biopsy and LEEP, and time interval from colposcopic directed biopsy to LEEP.

All colposcopic examinations and LEEP were performed by gynecologic oncologists. LEEP specimens were prepared by serial cutting in 2-3-millimeter thickness for pathological examination. The colposcopic directed biopsy and LEEP pathology were retrieved for review by a pathologist (AS) and final diagnosis was established with agreement of AS and the previous pathologist's report. If the final diagnosis of the colposcopic directed biopsy specimens was not CIN 2-3, they were excluded. Pathological discrepancy was defined as having CIN 2-3 in colposcopic directed biopsy specimens, but no dysplasia or having CIN 1 in LEEP specimens.

Post-LEEP follow-up using Pap test was performed every 6-12 months during the first 2 years. Patients who were lost to follow-up were excluded from the study. Persistent or recurrent disease was defined as having histological diagnosis of CIN 2-3 during the follow-up.

The sample size was calculated by using the single proportion formula, based on the prevalence of pathological discrepancy from the previous study which was $24 \%$ (Ryu et al., 2010; Witt et al., 2012; Rodriguez-Manfredi et al., 2013; Giannella et al., 2015; Nam et al., 2015; Zhang et al., 2015). Applying an acceptable error of 5\%, the sample size was 280 . Statistical analyses were performed using the Statistical Package for the Social Sciences software (IBM Corp., Armonk, NY, USA). Descriptive statistics were used to analyze clinical data of the patients. Associations between clinical factors and CIN 1 or less in LEEP specimens were analyzed using Chi-square test, Student- $t$ test and logistic regression analyses. Ap value of less than 0.05 was considered statistically significant.

\section{Results}

There were 280 patients with colposcopic directed biopsy-confirmed CIN 2-3 included in the study. Clinical characteristics are summarized in Table 1. Mean (SD) age of the patients was 35.8 (9.1) years. There were 125

Table 1. Clinical Characteristics of Enrolled Patients $(\mathrm{N}=280)$

\begin{tabular}{|c|c|}
\hline Characteristics & Value \\
\hline Age, mean $\pm \mathrm{SD}$ (years) & $35.8 \pm 9.1$ \\
\hline \multicolumn{2}{|l|}{ Parity } \\
\hline Nulliparous & $119(42.5)$ \\
\hline Multiparous & $161(57.5)$ \\
\hline \multicolumn{2}{|l|}{ Menopausal status } \\
\hline Premenopause & $256(91.4)$ \\
\hline Postmenopause & $24(8.6)$ \\
\hline \multicolumn{2}{|l|}{ Papanicolaou test } \\
\hline ASC-US & $61(21.8)$ \\
\hline LSIL & $64(22.8)$ \\
\hline HSIL & $110(39.3)$ \\
\hline ASC-H & $34(12.1)$ \\
\hline AGC & $1(0.4)$ \\
\hline Cancer & $10(3.6)$ \\
\hline \multicolumn{2}{|l|}{ Colposcopy } \\
\hline Satisfactory & $169(60.4)$ \\
\hline Unsatisfactory & $111(39.6)$ \\
\hline \multicolumn{2}{|l|}{ Colposcopic impression } \\
\hline Low grade & $111(39.6)$ \\
\hline High grade & $169(60.4)$ \\
\hline \multicolumn{2}{|c|}{ Colposcopic directed biopsy } \\
\hline CIN 2 & $115(41.1)$ \\
\hline CIN 3 & $165(58.9)$ \\
\hline \multicolumn{2}{|l|}{ LEEP results } \\
\hline No dysplasia & $30(10.7)$ \\
\hline CIN 1 & $41(14.7)$ \\
\hline CIN 2 & $32(11.4)$ \\
\hline CIN 3 & $177(63.2)$ \\
\hline AIS/cancer & $0(0)$ \\
\hline \multicolumn{2}{|l|}{ Margin } \\
\hline Free & $178(63.6)$ \\
\hline Involved by CIN 1 & $7(2.5)$ \\
\hline Involved by CIN 2-3 & $95(33.9)$ \\
\hline \multicolumn{2}{|c|}{ Time interval from biopsy to LEEP } \\
\hline 1 month & $104(37.1)$ \\
\hline 2 months & $95(33.9)$ \\
\hline$\geq 3$ months & $81(29.0)$ \\
\hline \multicolumn{2}{|c|}{ Recurrent/persistent disease } \\
\hline No & $264(94.3)$ \\
\hline CIN 1 & $13(4.6)$ \\
\hline CIN 2-3 & $3(1.1)$ \\
\hline
\end{tabular}

Values are presented as N (\%); ASC-US, atypical squamous cells of undetermined significance; LSIL, low grade squamous intraepithelial lesions; HSIL, high grade squamous intraepithelial lesions; ASC-H, atypical squamous cells cannot exclude HSIL; AGC, atypical glandular cells; CIN, cervical intraepithelial neoplasia; AIS, adenocarcinoma in situ; LEEP, loop electrosurgical excision procedure. 
Table 2. Clinical Characteristics of Patients According to Loop Electrosurgical Excision Procedure (LEEP) Pathology

\begin{tabular}{|c|c|c|c|}
\hline \multirow[b]{2}{*}{ Characteristics } & \multicolumn{2}{|c|}{ LEEP pathology } & \multirow[b]{2}{*}{ p-value } \\
\hline & No dysplasia/CIN $1(\mathrm{~N}=71)$ & CIN 2-3 $(\mathrm{N}=209)$ & \\
\hline Age, mean \pm SD (years) & $32.1 \pm 7.1$ & $37.1 \pm 9.3$ & $<0.001$ \\
\hline \multicolumn{4}{|l|}{ Parity } \\
\hline Nulliparous & $45(63.4)$ & $74(35.4)$ & \multirow[t]{2}{*}{$<0.001$} \\
\hline Multiparous & $26(36.6)$ & 135 (64.6) & \\
\hline \multicolumn{4}{|l|}{ Menopausal status } \\
\hline Premenopause & $65(91.5)$ & $191(91.4)$ & \multirow[t]{2}{*}{0.970} \\
\hline Postmenopause & $6(8.5)$ & $18(8.6)$ & \\
\hline \multicolumn{4}{|l|}{ Papanicolaou test } \\
\hline Low grade ${ }^{a}$ & $54(76.1)$ & $71(34.0)$ & \multirow[t]{2}{*}{$<0.001$} \\
\hline High grade ${ }^{b}$ & $17(23.9)$ & $138(66.0)$ & \\
\hline \multicolumn{4}{|l|}{ Colposcopy } \\
\hline Satisfactory & $37(52.1)$ & $132(63.2)$ & \multirow[t]{2}{*}{0.100} \\
\hline Unsatisfactory & $34(47.9)$ & $77(36.8)$ & \\
\hline \multicolumn{4}{|l|}{ Colposcopic impression } \\
\hline Low grade & $60(84.5)$ & $51(24.4)$ & \multirow[t]{2}{*}{$<0.001$} \\
\hline High grade & $11(15.5)$ & $158(75.6)$ & \\
\hline \multicolumn{4}{|c|}{ Colposcopic directed biopsy } \\
\hline CIN 2 & $55(77.5)$ & $60(28.7)$ & \multirow[t]{2}{*}{$<0.001$} \\
\hline CIN 3 & $16(22.5)$ & $149(71.3)$ & \\
\hline \multicolumn{4}{|c|}{ Recurrent/persistent disease } \\
\hline $\mathrm{No} / \mathrm{CIN} 1$ & $71(100.0)$ & $206(98.6)$ & \multirow[t]{2}{*}{0.310} \\
\hline CIN 2-3 & $0(0)$ & $3(1.4)$ & \\
\hline \multicolumn{4}{|c|}{ Time interval from biopsy to LEEP } \\
\hline 1 month & $17(24.0)$ & $87(41.6)$ & \multirow[t]{3}{*}{$<0.001$} \\
\hline 2 months & $29(40.8)$ & $66(31.6)$ & \\
\hline$\geq 3$ months & $25(35.2)$ & $56(26.8)$ & \\
\hline
\end{tabular}

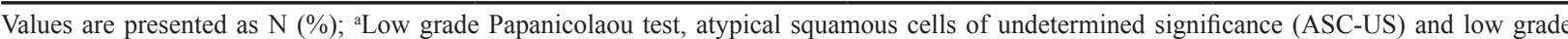
squamous intraepithelial lesions (LSIL); 'High grade Papanicolaou test, high grade squamous intraepithelial lesions (HSIL); atypical squamous cells cannot exclude HSIL (ASC-H); atypical glandular cells (AGC) and cancer; CIN, cervical intraepithelial neoplasia.

(44.6\%) patients with low grade Pap results, including atypical squamous cells of undetermined significance (ASC-US) and low grade squamous intraepithelial lesion (LSIL) and 155 (55.4\%) patients with high grade Pap results, including high grade squamous intraepithelial lesion (HSIL), atypical squamous cells cannot exclude HSIL (ASC-H), atypical glandular cells (AGC), squamous cell carcinoma and adenocarcinoma. Histological diagnosis from colposcopic directed biopsy specimens were CIN 2 in 115 (41.1\%) patients and CIN 3 in 165 (58.9\%) patients. LEEP histologic results were 30 (10.7\%) patients with no dysplasia, 41 (14.7\%) patients with CIN 1, $32(11.4 \%)$ patients with CIN 2 and 177 (63.2\%) patients with CIN 3. Neither adenocarcinoma in situ (AIS) nor cancer was detected in LEEP specimens.

Predictors of CIN 1 or less in LEEP specimens

Pathological discrepancy was found in 71 out of 280

Table 3. Multivariate Logistic Regression Analyses of Risk Factors for Predicting an Absence of High Grade Dysplasia in Loop Electrosurgical Excision Procedure (LEEP) Specimens

\begin{tabular}{lcccc}
\hline & \multicolumn{3}{c}{$95 \%$ confidence interval } \\
Risk factors & OR & Lower & Upper & p-value \\
\hline Age & 0.959 & 0.882 & 1.042 & 0.326 \\
Parity: nulliparity & 3.375 & 1.245 & 9.150 & 0.017 \\
Papanicolaou test: low grade & 6.410 & 2.877 & 14.280 & $<0.001$ \\
Colposcopic impression: low grade & 16.509 & 5.844 & 46.632 & $<0.001$ \\
Colposcopic directed biopsy: CIN 2 & 1.351 & 0.344 & 5.303 & 0.666 \\
Time interval from biopsy to LEEP & 0.678 & 0.217 & 2.118 & 0.504 \\
\hline
\end{tabular}


Table 4. Sensitivity Analyses of Risk Factors for Predicting an Absence of High-grade CIN in LEEP Specimens

\begin{tabular}{lcccc}
\hline Risk factors & Sensitivity (\%) & Specificity (\%) & PPV (\%) & NPV (\%) \\
\hline Nulliparity & 63 & 64 & 37 & 83 \\
Low grade Pap test & 76 & 66 & 43 & 89 \\
Low grade colposcopic impression & 85 & 76 & 54 & 93 \\
\hline
\end{tabular}

PPV, positive predictive value; NPV, negative predictive value; CIN, cervical intraepithelial neoplasia; LEEP, loop electrosurgical excision procedure

patients $(25.4 \%)$. Risk factors for having pathological discrepancy are presented in Table 2. Increased age, nulliparity, low grade Pap results, low grade colposcopic impression, CIN 2 on colposcopic directed biopsy and increased time interval between biopsy and LEEP were significantly associated with CIN 1 or less in LEEP specimens. Multivariate logistic regression analyses showed that nulliparity, low grade Pap results and low grade colposcopic impression were associated with CIN 1 or less in LEEP specimens [OR $(95 \% \mathrm{CI}): 3.375$ $(1.245-9.150), p=0.017 ; 6.410(2.877-14.280), p<0.001$ and 16.509 (5.844-46.632), $\mathrm{p}<0.001$, respectively] (Table 3).

Sensitivity, specificity, positive predictive value (PPV) and negative predictive value (NPV) of nulliparity, low grade Pap results and low grade colposcopic impression for predicting the absence of high-grade CIN in LEEP specimens are shown in Table 4. Low grade colposcopic impression was found to be the most sensitive ( $85 \%$ ) and specific $(76 \%)$.

\section{Follow-up data}

Median (range) follow-up time of patients who had LEEP specimens with CIN 1 or less $(\mathrm{N}=71)$ and CIN 2-3 $(\mathrm{N}=209)$ were 10 (6-17) months and 12 (7-14) months, respectively.

Out of 71 patients with CIN 1 or less in LEEP specimens, $1(1.4 \%)$ had abnormal cervical cytology with followed-up colposcopic directed biopsy not found CIN 2-3. Among 209 patients with CIN 2-3 in LEEP specimens, 7 (3.3\%) and $19(9.1 \%)$ had abnormal cervical cytology defined as low grade and high grade, respectively. Out of 26 patients with abnormal cervical cytology, 3 patients had CIN 2-3. Therefore, persistent or recurrent CIN 2-3 was diagnosed in $3(1.4 \%)$ of 26 patients with CIN 2-3 in LEEP specimens. However, no persistent or recurrent CIN 2-3 disease was detected in patients with CIN 1 or less in LEEP specimens.

\section{Discussion}

Current recommendations by the American Society for Colposcopy and Cervical Pathology (ASCCP) suggest that patients with CIN 2-3 diagnosed by colposcopic directed biopsy should be treated with excision or ablation (Massad et al., 2013). The reasons for the recommendations are lower rate of spontaneous regression and risk of progression to cancer especially CIN 3 lesions (Massad et al., 2013). LEEP is one of the excisional methods for the treatment of these lesions. The minority of patients with CIN 2-3 on colposcopic directed biopsy have no dysplasia or have only CIN
1 in subsequent LEEP specimens. This study showed $25.4 \%$ of CIN 1 or less in LEEP specimens of patients with colposcopic directed biopsy-confirmed CIN 2-3. Previous studies have reported the prevalence of $14-24 \%$ of pathological discrepancy (Ryu et al., 2010; Witt et al., 2012; Rodriguez-Manfredi et al., 2013; Giannella et al., 2015; Nam et al., 2015; Zhang et al., 2015; Noothong et al., 2017). The rates of pathological discrepancy varied because of differences in studied population and definition of pathological discrepancy among studies. This study defined pathological discrepancy as the diagnosis of CIN 2-3 in colposcopic directed biopsy specimens, but identification of CIN 1 or less in LEEP specimens. On the other hand, some studies defined pathological discrepancy as only an absence of dysplasia found in LEEP specimens in patients with colposcopic directed biopsy-confirmed CIN 2-3 (Ryu et al., 2010; Nam et al., 2015). Absence of high grade CIN in LEEP specimens despite having high grade CIN in colposcopic directed biopsy specimens might be caused by several reasons. First, CIN lesion was small and it was completely removed by the biopsy procedure ( $\mathrm{Li}$ et al., 2009). Second, misdiagnosis of LEEP specimens or high grade CIN was not removed by LEEP (Ryu et al., 2010), Third, the remaining lesion might spontaneously regress (Melnikow et al., 1998; Li et al., 2009).

Multivariate logistic regression analyses of this study found that nulliparity, low grade Pap results and low grade colposcopic impression were the predicting factors of having CIN 1 or less in LEEP specimens. However, there was no association between CIN 2 in colposcopic directed biopsy specimens and CIN 1 or less in LEEP specimens. This finding was different from that of most previous studies which have shown CIN 2 in colposcopic directed biopsy specimens was the predicting factor of having CIN 1 or less in LEEP specimens (Giannella et al., 2015; Nam et al., 2015; Zhang et al., 2015; Noothong et al., 2017). Our study found that low grade colposcopic impression was a predicting factor of having CIN 1 or less in LEEP specimens. This finding was in agreement with the study of Giannella et al., (2015) in which they demonstrated that CIN 2 on cervical biopsy and low grade colposcopic impression were predictors of minor cone histology. In addition, study of prognostic value of colposcopic impression showed that patients with low grade Pap results and normal colposcopic impression had a low risk of having high grade CIN within 3 years (Cruickshank et al., 2015).

The follow-up data of this study showed no persistent or recurrent CIN 2-3 in patients who had CIN 1 or less in LEEP specimens. However, this result was different from that of the study by Giannella et al., (2015). Giannella et 
al., (2015) reported that recurrence rates of high grade CIN were $7.4 \%$ and $10.6 \%$ in patients who had CIN 1 or less and CIN 2-3 in LEEP specimens, respectively. Therefore, patients with both CIN 2-3 and CIN 1 or less in LEEP specimens following a biopsy diagnosis of CIN 2-3 should be similarly followed up.

Regarding fertility-sparing approach for preservation of reproductive potential of the patients, the impact of treatment on quality of life and psychological issue of these patients should be considered (Vitale et al., 2017; Chiofalo et al., 2017).

The strength of this study was that all histologic slides of cervical biopsy and LEEP were reviewed by a pathologist. However, there were some limitations, including being a retrospective design, and lack of data on size and position of cervical lesions, size of LEEP specimens and Human papillomavirus (HPV) testing results. HPV testing was not performed in a majority of patients enrolled in this study.

Several biomarkers such as p16ink4a, p16 and Ki67, which were demonstrated to be predictors of intraepithelial lesions, have more chance to develop to invasive forms (Vitale et al., 2016; Valenti et al., 2017; Nicol et al., 2018). In addition, p16ink4a immunohistochemistry was presented in low grade lesions associated with high risk HPV types which have a high risk of progression (Vitale et al., 2016; Valenti et al., 2017). Furthermore, p16 immunostaining was shown to reduce the frequency of negative LEEP after CIN 2-3 diagnoses of cervical colposcopic biopsies (de Sam Lazaro et al., 2016). However, our study did not investigate these specific biomarkers.

In conclusion, approximately $25 \%$ of patients with CIN 2-3 in colposcopic directed biopsy specimens had CIN 1 or less found in LEEP specimens. Nulliparity, low grade Pap results and low grade colposcopic impression were predicting factors of CIN 1 or less in LEEP specimens. Although LEEP is a safe procedure, it may carry a risk of future reproductive problems (Vitale et al., 2017). These findings could be useful for guiding treatment options and reducing unnecessary LEEP in patients with colposcopic directed biopsy-confirmed CIN 2-3 who had low grade Pap results and low grade colposcopic impression. Therefore, conservative treatment may be another option for patients who have these predicting factors.

\section{Acknowledgements}

The authors thank Thammasat University Hospital for helping with acquisition of patients' data. We gratefully acknowledge the financial support provided by Thammasat University Research Fund under the TU Research Scholar, Contract No TP 1/4/2561.

\section{References}

Chiofalo B, Palmara V, Lagana AS, et al (2017). Fertility sparing surgery for stage IA type I and G2 endometrial cancer in reproductive-aged patients: evidence-based approach and future perspectives. Updates Surg, 69, 29-34.

Cruickshank ME, Cotton SC, Sharp L, et al (2015). Management of women with low grade cytology: how reassuring is a normal colposcopy examination?. BJOG, 122, 380-6.

de Sam Lazaro S, Newbill CP, Berlin M, Morgan TK (2016). p16 staining of cervical biopsies may decrease the frequency of unnecessary loop electrosurgical excision procedures. J Low Genit Tract Dis, 20, 201-6.

Eduardo AM, Dinh TV, Hannigan EV, Yandell RB, Schnadig VJ (1996). Outpatient loop electrosurgical excision procedure for cervical intraepithelial neoplasia. Can it replace cold knife conization?. J Reprod Med, 41, 729-32.

Giannella L, Mfuta K, Gardini G, et al (2015). High-grade $\mathrm{CIN}$ on cervical biopsy and predictors of the subsequent cone histology results in women undergoing immediate conization. Eur J Obstet Gynecol Reprod Biol, 186, 68-74.

Kyrgiou M, Tsoumpou I, Vrekoussis T, et al (2006). The up-to-date evidence on colposcopy practice and treatment of cervical intraepithelial neoplasia: the Cochrane colposcopy \& cervical cytopathology collaborative group (C5 group) approach. Cancer Treat Rev, 32, 516-23.

Li ZG, Qian de Y, Cen JM, Chen GD, Shu YH (2009). Three-step versus "see-and-treat" approach in women with high-grade squamous intraepithelial lesions in a low-resource country. Int J Gynaecol Obstet, 106, 202-5.

Martin-Hirsch PP, Paraskevaidis E, Bryant A, Dickinson HO (2013). Surgery for cervical intraepithelial neoplasia. Cochrane Database Syst Rev, 12, CD001318.

Massad LS, Einstein MH, Huh WK, et al (2013). 2012 updated consensus guidelines for the management of abnormal cervical cancer screening tests and cancer precursors. J Low Genit Tract Dis, 17, S1-S27.

Melnikow J, Nuovo J, Willan AR, Chan BK, Howell LP (1998). Natural history of cervical squamous intraepithelial lesions: a meta-analysis. Obstet Gynecol, 92, 727-35.

Nam K, Ryu A, Jeon S, et al (2015). Clinical significance of a negative loop electrosurgical excision procedure biopsy in patients with biopsy-confirmed high-grade cervical intraepithelial neoplasia. J Low Genit Tract Dis, 19, 103-9.

Nicol AF, de Andrade CV, Gomes SC Jr, et al (2018). The distribution of novel biomarkers in carcinoma-in-situ, microinvasive, and squamous cell carcinoma of the uterine cervix. Ann Diagn Pathol, 38, 115-22.

Noothong S, Inthasorn P, Warnnissorn M (2017). Pathological discrepancy between colposcopic directed cervical biopsy and Loop Electrosurgical-Excision Procedures (LEEPs) in patients with biopsies proven high grade cervical intraepithelial neoplasia. Taiwan J Obstet Gynecol, 56, 628-31.

Pinto AP, Crum CP (2000). Natural history of cervical neoplasia: defining progression and its consequence. Clin Obstet Gynecol, 43, 352-62.

Rodriguez-Manfredi A, Alonso I, delPino M, et al (2013). Predictors of absence of cervical intraepithelial neoplasia in the conization specimen. Gynecol Oncol, 128, 271-6.

Ryu A, Nam K, Chung S, et al (2010). Absence of dysplasia in the excised cervix by a loop electrosurgical excision procedure in the treatment of cervical intraepithelial neoplasia. J Gynecol Oncol, 21, 87-92.

Soutter WP, Sasieni P, Panoskaltsis T (2006). Long-term risk of invasive cervical cancer after treatment of squamous intraepithelial neoplasia. Int J Cancer, 118, 2048-55.

Valenti G, Vitale SG, Tropea A, Biondi A, Lagana AS (2017). Tumor markers of uterine cervical cancer: a new scenario to guide surgical practice?. Updates Surg, 69, 441-9.

Vitale SG, La Rosa VL, Rapisarda AMC, Lagana AS (2017). The importance of fertility preservation counseling in patients with gynecologic cancer. $J$ Reprod Infertil, 18, 261-3.

Vitale SG, Rossetti D, Tropea A, Biondi A, Lagana AS (2017). 
Yenrudee Poomtavorn et al

Fertility sparing strategies in patients affected by placental site trophoblastic tumor. Curr Treat Options Oncol, 18, 58.

Vitale SG, Valenti G, Rapisarda AMC, et al (2016). P16INK4a as a progression/regression tumour marker in LSIL cervix lesions: Our clinical experience. Eur J Gynaecol Oncol, 37, 685-8.

Witt BL, Factor RE, Jarboe EA, Layfield LJ (2012). Negative loop electrosurgical cone biopsy finding following a biopsy diagnosis of high-grade squamous intraepithelial lesion: frequency and clinical significance. Arch Pathol Lab Med, 136, 1259-61.

Zhang L, Li Q, Zhao M, Jia L, Zhang Y (2015). Discrepancies between biopsy-based and excision-based grading of cervical intraepithelial neoplasia: the important role of time between excision and biopsy. Int J Gynecol Pathol, 34, 221-7.

This work is licensed under a Creative Commons AttributionNon Commercial 4.0 International License. 